Draft Version July 9, 2018

Preprint typeset using $\mathrm{AT}_{\mathrm{E} X} \mathrm{X}$ style emulateapj v. 11/10/09

\title{
POSSIBLE DETECTION OF THE STELLAR DONOR OR REMNANT FOR THE TYPE Iax SUPERNOVA 2008ha
}

\author{
Ryan J. Foley ${ }^{1,2}$, Curtis McCully ${ }^{3}$, Saurabh W. Jha ${ }^{3}$, Lars Bildsten ${ }^{5,4}$, Wen-Fai Fong ${ }^{6}$, Gautham Narayan $^{7}$, \\ Armin Rest ${ }^{7}$, And Maximilian D. Stritzinger ${ }^{9}$
}

Draft version July 9, 2018

\begin{abstract}
Type Iax supernovae (SNe Iax) are thermonuclear explosions that are related to SNe Ia, but are physically distinct. The most important differences are that SNe Iax have significantly lower luminosity $\left(1 \%-50 \%\right.$ that of typical SNe Ia), lower ejecta mass $\left(\sim 0.1-0.5 \mathrm{M}_{\odot}\right)$, and may leave a bound remnant. The most extreme SN Iax is SN 2008ha, which peaked at $M_{V}=-14.2 \mathrm{mag}$, about 5 mag below that of typical SNe Ia. Here, we present Hubble Space Telescope (HST) images of UGC 12682, the host galaxy of SN 2008ha, taken 4.1 years after the peak brightness of SN 2008ha. In these deep, high-resolution images, we detect a source coincident (0.86 HST pixels; $\left.0.043^{\prime \prime} ; 1.1 \sigma\right)$ with the position of SN 2008ha with $M_{\mathrm{F} 814 \mathrm{~W}}=-5.4 \mathrm{mag}$. We determine that this source is unlikely to be a chance coincidence, but that scenario cannot be completely ruled out. If this source is directly related to SN 2008ha, it is either the luminous bound remnant of the progenitor white dwarf or its companion star. The source is consistent with being an evolved $>3 \mathrm{M}_{\odot}$ initial mass star, and is significantly redder than the SN Iax 2012Z progenitor system, the first detected progenitor system for a thermonuclear SN. If this source is the companion star for SN 2008ha, there is a diversity in SN Iax progenitor systems, perhaps related to the diversity in SN Iax explosions. If the source is the bound remnant of the white dwarf, it must have expanded significantly. Regardless of the nature of this source, we constrain the progenitor system of SN 2008ha to have an age of $<80$ Myr.
\end{abstract}

Subject headings: galaxies - individual(UGC 12682), supernovae - general, supernovae -individual (SN 2008ha)

\section{INTRODUCTION}

Type Iax supernovae (SNe Iax) are a class of thermonuclear stellar explosions physically distinct from SNe Ia, but sharing several characteristics (see Jha et al. 2006 and Foley et al. 2013 for reviews). This class is the most common of all types of peculiar SNe by both number and rate, occurring at roughly $30 \%$ the rate of SNe Ia (Foley et al. 2013). All observational properties indicate that these explosions are less energetic than SNe Ia (e.g., Li et al. 2003; (Phillips et al. 2007; Foley et al. 2013).

While the progenitor systems of $\mathrm{SNe}$ Ia are still uncertain (see Maoz et al. 2013 for a review), a reasonable progenitor model and explosion mechanism that explains most SN Iax observations has emerged. As the number of observations has increased (Foley et al. 2009, 2010a, b, 2013; Valenti et al.

1 Astronomy Department, University of Illinois at UrbanaChampaign, 1002 W. Green Street, Urbana, IL 61801, USA

2 Department of Physics, University of Illinois UrbanaChampaign, 1110 W. Green Street, Urbana, IL 61801, USA

3 Department of Physics and Astronomy, Rutgers, The State University of New Jersey, 136 Frelinghuysen Road, Piscataway, NJ 08854, USA

5 Kavli Institute for Theoretical Physics, Santa Barbara, CA 93106, USA

4 Department of Physics, University of California, Santa Barbara, CA 93106, USA

6 Harvard-Smithsonian Center for Astrophysics, 60 Garden Street, Cambridge, MA 02138, USA

7 National Optical Astronomy Observatory, 950 North Cherry Avenue, Tucson, AZ 85719-4933, USA

8 Space Telescope Science Institute, 3700 San Martin Drive, Baltimore, MD 21218, USA

9 Department of Physics and Astronomy, Aarhus University, Ny Munkegade, DK-8000 Aarhus C, Denmark
2009; Maund et al. 2010; McClelland et al. 2010; Milne et al. 2010; Narayan et al. 2011; Kromer et al. 2013; McCully et al. 2014a; Stritzinger et al. 2014), the best model for these SNe continues to be a deflagration of a $\mathrm{C} / \mathrm{O}$ white dwarf (WD) that does not burn through the entire WD as first proposed by Foley et al. (2009). Because of He seen in the spectra of two SNe Iax and their implied delay time of $\lesssim 500$ Myr, Foley et al. (2013) expanded on this initial model by employing a He-star companion, similar to a previously suggested progenitor system for SNe Ia (Iben \& Tutukov 1991; Liu et al. 2010). Observations suggest, and this model implies, that at least some SNe Iax do not unbind their progenitor star, leaving behind a remnant (Folev et al. 2009, 2010a, 2013; Jordan et al. 2012; Kromer et al. 2013; McCully et al. 2014a). This model can explain all observational properties of SNe Iax except perhaps the low polarization seen in a single SN Iax Chornock et al. 2006; Maund et al. 2010).

This model has been directly tested by pre-explosion Hubble Space Telescope (HST) images of SN 2008ge (Foley et al. 2010b) and SN 2012Z (McCully et al., in press; hereafter M14). No progenitor was detected in the relatively shallow images for SN 2008ge, but the data excluded most massive stars as the progenitor (as suggested by Valenti et al. 2009 and Moriva et al. 2010). Deep limits on the star formation for the host galaxy of SN $2008 \mathrm{ge}$ also indicated that SN 2008ge did not have a massive progenitor star (Foley et al. 2010b).

M14 detected a star in pre-explosion HST images at the position of SN 2012Z. This was the first direct detection of a thermonuclear SN progenitor system. This relatively 


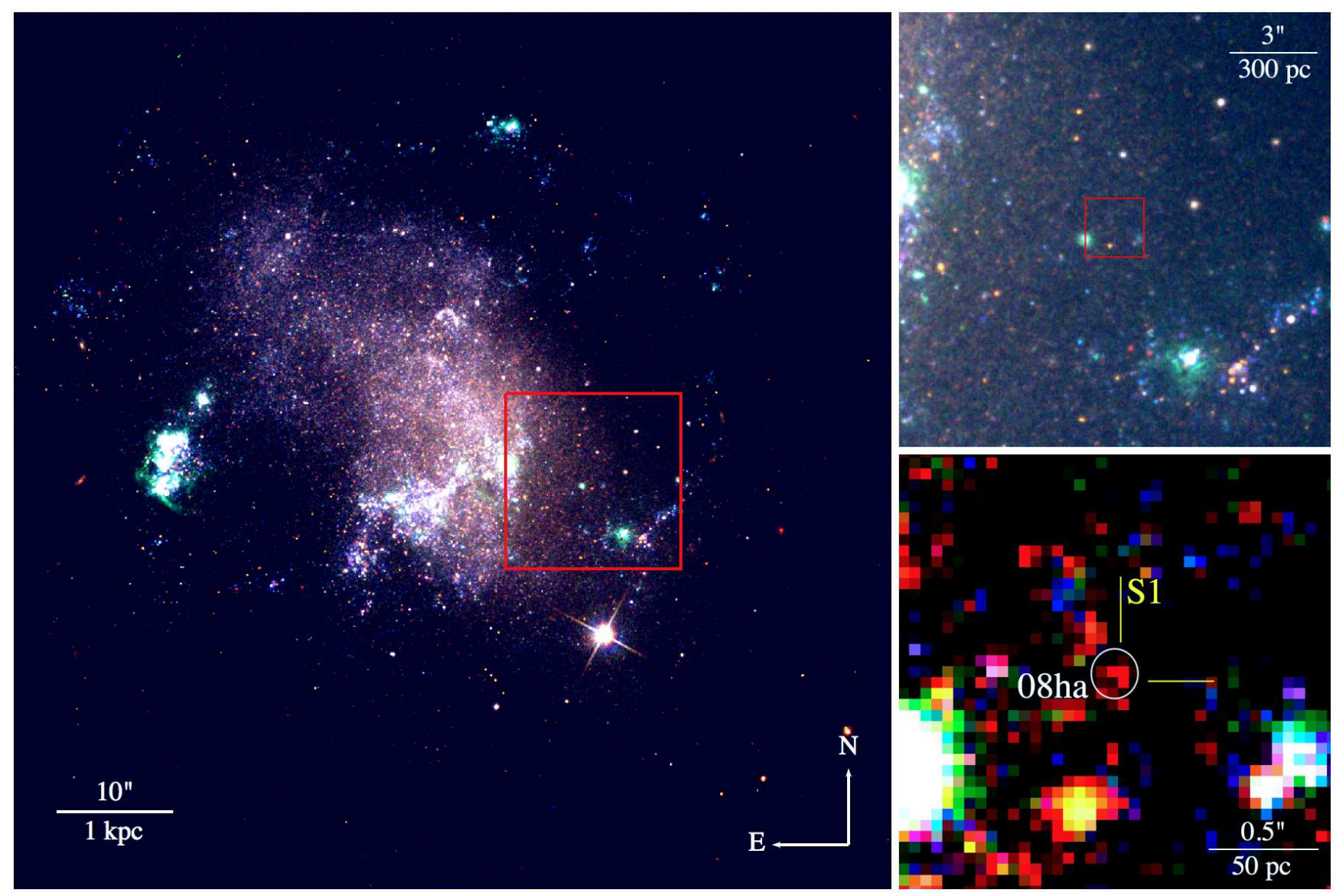

FIG. 1. - False-color HST image of UGC 12682, the host galaxy of SN 2008ha. The red, green, and blue channels correspond to F814W F625W, and F435W, respectively. Left: $75^{\prime \prime} \times 75^{\prime \prime}$ image. The red box is $15^{\prime \prime} \times 15^{\prime \prime}$ and centered on the position of SN 2008 ha. Top-Right: $15^{\prime \prime} \times 15^{\prime \prime}$ image corresponding to the red box in the left panel. The red box is $2^{\prime \prime} \times 2^{\prime \prime}$. Bottom-Right: $2^{\prime \prime} \times 2^{\prime \prime}$ image corresponding to the red box in the top-right panel. A $3 \sigma$ ellipse is centered on the position of SN 2008ha. There is a single source near the center of the ellipse, which we label "S1." The bright source to the south-east of S1 is an H II region with a measured metallicity of $12+\log [\mathrm{O} / \mathrm{H}]=8.16$ (Folev et al. 2009).

luminous star is most consistent with a massive $(M \approx$ $2 \mathrm{M}_{\odot}$ at the time of explosion) He star, as predicted by Foley et al. (2013).

In this manuscript, we present post-explosion $H S T$ images of the host galaxy of SN 2008ha, the least luminous SN Iax (Folev et al. 2009, 2010a; Valenti et al. 2009; Stritzinger et al. 2014). SN 2008ha was discovered on 2008 November 7.17 (UT dates are used throughout this paper; Puckett et al. 2008) in UGC 12682, an irregular galaxy with a recession velocity of $1393 \mathrm{~km} \mathrm{~s}^{-1}$, corresponding to a Virgo-infall corrected distance modulus of $\mu=31.64 \pm 0.15 \mathrm{mag}$ and $D=21.3 \pm 1.5 \mathrm{Mpc}$, assuming $H_{0}=73 \pm 5 \mathrm{~km} \mathrm{~s}^{-1} \mathrm{Mpc}^{-1}$. Foley (2008) first classified SN 2008ha as a SN Iax, but also noted its extreme characteristics and the possibility that SN 2008ha may not have unbound its progenitor star.

In the post-explosion images, we detect a point source near the position of SN 2008ha. We outline the data acquisition and reduction in Section 2 In Section 3. we detail the properties of this source, and examine several possibilities for its nature including being a chance coincidence, SN emission, the stellar remnant, and the companion star from the progenitor system. We also constrain the properties of the progenitor system and remnant if the source is unrelated to SN 2008ha. We discuss the implications of our findings and summarize our conclusions in Section 4 .

\section{OBSERVATIONS AND DATA REDUCTION}

UGC 12682 was observed with HST/ACS on 2012 January 2.2 (Program GO-12999; PI Foley). The full observing sequence obtained 1164, 764, 840, and 1240 seconds in the F435W, F555W, F625W, and F814W filters (corresponding roughly to $B V R I)$.

We combined exposures (including cosmic ray rejection) using AstroDrizzle. We register the individual flatfielded (flt) frames using TweakReg from the DrizzlePac package. We registered the absolute astrometry to ground-based images of the same field using the $\mathrm{F} 555 \mathrm{~W}$ exposures. We then tied the relative astrometry of the other filters to this image. The typical rms of the relative astrometry solution was $0.005^{\prime \prime}=0.1$ ACS pixels. We drizzle the images to the native scale of ACS, $0.05^{\prime \prime}$ per pixel. A false-color image of UGC 12682 and zoomed panels of the region near the position of SN 2008ha are presented in Figure 1.

We photometered the HST images using the PSF photometry software DolPhot, an extension of HSTPhot (Dolphin 2000). DolPhot photometers individual flt frames and then combines the photometry. We use the 
suggested parameters for ACS from the Dolphot manual. We only consider photometry with a "flag" of 0 and an "object type" of 1 (point sources) in our results.

Using an $r$-band image taken with the 1.0-m Swope telescope, we were able to determine the astrometric position of SN 2008ha in the HST images. The Swope image, from which the Stritzinger et al. (2014) SN 2008ha photometry was derived, had a template image subtracted for the region around SN 2008ha. This provides a precise measurement of the position of SN 2008ha relative to field stars without contamination from underlying emission (particularly from a nearby H II region).

The Swope and HST images had a total of 14 stars in common with well-measured positions. Following the procedures of Folev et al. (2010b, and references therein), we determined a geometric transformation between the two images. However, tests showed that inclusion of stars far from SN 2008ha adversely affected the accuracy of the transformation as determined by looking at positions of stars not included in the fitting. The poor accuracy is likely because of higher-order distortions on large spatial scales that could not be adequately removed with the limited number of stars. However, we found that using only the 8 stars closest to SN 2008ha removed all obvious bias near the location of SN 2008ha.

Using these 8 stars, the accuracy of the geometric transformation is robust near the SN position. However, the nominal uncertainties in the SN position $(\sim 0.4 \mathrm{ACS}$ pixels; $\sim 0.02^{\prime \prime}$ ) may be underestimated given the previously determined errors. To provide a better estimate of the positional uncertainty, we re-determined the position of SN 2008ha 100 times by selecting bootstrapped samples (with replacement) from the initial sample of 8 stars. We performed this analysis several times with varying orders for the polynomial transformations. Using outlier-resistant statistics, the average SN 2008ha position from the bootstrapped samples are consistent with our best estimate. We use the outlier-resistant scatter of the resulting positions, 0.44 and 0.50 ACS pixels in the R.A. and Dec. directions, respectively, which are larger than the nominal uncertainty from the nominal position, as the uncertainty in the position of SN 2008ha from the geometric transformation. To this, we add in quadrature the statistical uncertainty in the position of SN 2008ha in the Swope image, which is equivalent to 0.08 ACS pixels, and the estimated systematic uncertainty in determining the SN position, which is equivalent to 0.44 ACS pixels. The total uncertainty in measuring the position of SN 2008ha in the HST images is thus 0.71 and 0.75 ACS pixels $\left(0.037^{\prime \prime}\right.$ and $\left.0.039^{\prime \prime}\right)$ in the R.A. and Dec. directions, respectively.

Offset by 0.86 ACS pixels, corresponding to $0.043^{\prime \prime}$, from the SN position, there is a source clearly detected in the $\mathrm{F} 814 \mathrm{~W}$ band $(\mathrm{S} / \mathrm{N}=6.9)$. This source is marginally detected in the $\mathrm{F} 625 \mathrm{~W}$ band $(\mathrm{S} / \mathrm{N}=1.8)$, and undetected in the bluer bands. We label this source "S1." The uncertainty in the position of this source is roughly 0.4 ACS pixels. Adding this in quadrature to the uncertainty in the position of SN 2008ha, S1 is $1.1 \sigma$ from the position of SN 2008ha. Photometry of S1 yields magnitudes of $<27.7,<27.3,28.1 \pm 0.6$, and $26.34 \pm 0.16 \mathrm{mag}$ in the HST filters, from blue to red, respectively, with the limits being $3-\sigma$ limits.

The $\mathrm{F} 625 \mathrm{~W}-\mathrm{F} 814 \mathrm{~W}$ color of $\mathrm{S} 1$ is redder than almost all nearby stars. Of the 100 stars closest to the position of SN 2008ha (including S1), 84 are bluer, and 13 have non-detections in F625W, but are fainter in F814W such that they could be bluer than S1. That is, only 2 of the closest 100 stars are definitely redder than S1.

Assuming our nominal distance modulus and correcting for Milky Way reddening of $E(B-V)=0.07 \mathrm{mag}$ (Schlegel et al. 1998; Schlafly \& Finkbeiner 2011), we can derive the luminosity of S1. Fitting a blackbody to the photometry of S1 (assuming no host-galaxy reddening), we find a best-fit temperature of $2100 \pm 500 \mathrm{~K}$ and a radius of $1500 \pm 800 R_{\odot}$, and a bolometric luminosity of $L_{\mathrm{bol}}=1.5 \times 10^{38} \mathrm{erg} \mathrm{s}^{-1}$. Using this temperature and the F814W absolute magnitude, we place S1 on a Hertzsprung-Russell (HR) diagram (Figure 2).

Although it is difficult to measure host-galaxy reddening from SN Iax colors (Foley et al. 2013), SN 2008ha did not appear to have any host-galaxy reddening (Foley et al. 2009). The position of the SN in its host galaxy also makes a substantial amount of dust in the interstellar medium unlikely. However, it is possible that there is newly formed circumstellar dust.

V445 Pup, a helium nova that ejected $\sim 10^{-4} \mathrm{M}_{\odot}$ of material formed a significant amount of circumstellar dust 7.5 months after discovery (Kato et al. 2008). Under the right conditions, SN 2008ha could have generated a similar amount of dust, which could also account for the very red colors of S1. Unfortunately, we cannot constrain the extinction from newly formed circumstellar dust with our current observations. Ignoring the possibility of circumstellar dust, the colors and luminosity of S1 are consistent with an thermal-pulsing asymptotic giant branch (TP-AGB) star that had an initial mass of $>3 \mathrm{M}_{\odot}$ (see Section 3.4).

\section{ANALYSIS}

Having identified a source that is spatially coincident with SN 2008ha, we now examine its physical properties in detail. There are four scenarios for this source: it is unrelated to SN 2008ha, it is emission from the SN itself, it is the luminous remnant of the WD progenitor, or it is the companion star to the WD.

Additionally, we examine constraints on the progenitor system and remnant if S1 is not associated with SN 2008ha.

\subsection{Chance Coincidence}

There are many bright, resolved stars in our images of UGC 12682. For certain positions, there will be a chance superposition with one of these stars. As noted above, $\mathrm{S} 1$ is $0.86 \mathrm{ACS}$ pixels, equivalently $0.043^{\prime \prime}$ and $1.1 \sigma$, from the position of SN 2008ha. We now calculate the probability of such a similar chance alignment.

Our analysis follows that of M14. Within a $40 \times$ 320 pixel $\left(2^{\prime \prime} \times 16^{\prime \prime}\right)$ box centered on SN 2008 ha, we detect 89 stars with $\mathrm{S} / \mathrm{N}>3$ in at least one of our $H S T$ images. The dimensions of the box were chosen such that the major axis was roughly perpendicular to the stellar density gradient, and the density of stars within this box should be roughly representative of the stellar density at the position of SN 2008ha.

Given our astrometric uncertainty, there is a $0.90 \%$ (7.0\%) chance that a random position near SN 2008ha would be within $1.1 \sigma(3 \sigma)$ of any detected star (in any of 


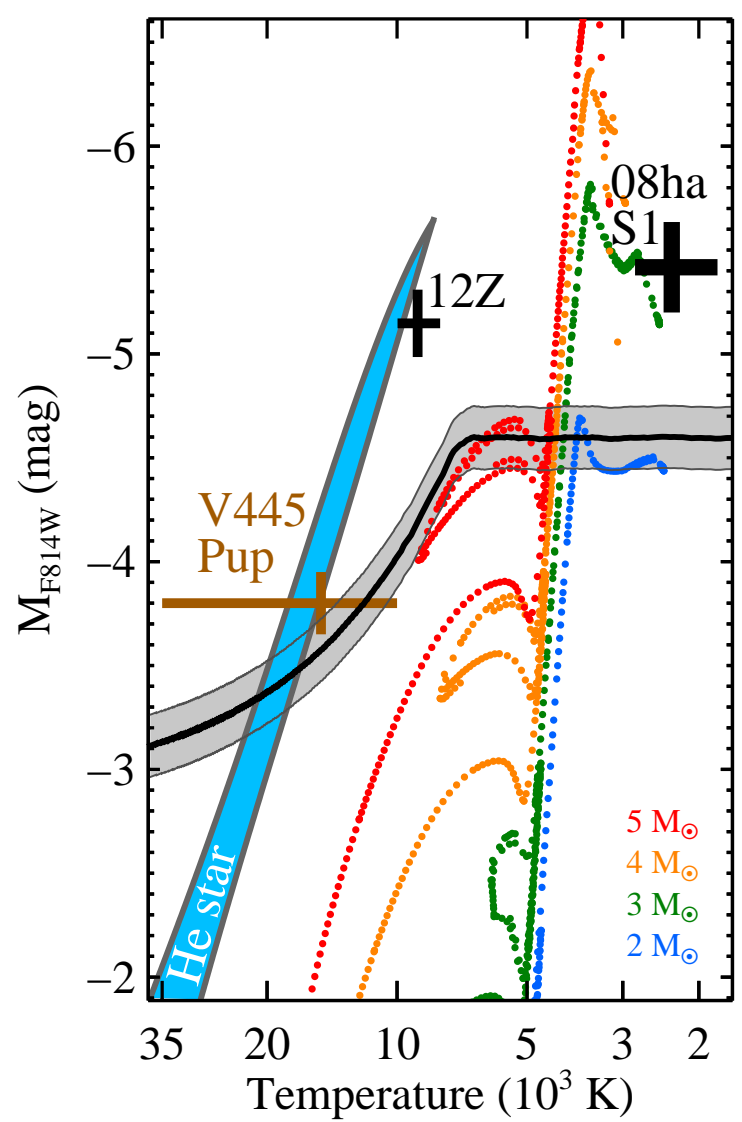

FIG. 2.- Hertzsprung-Russell diagram for S1 (thick black cross; $2100 \pm 500 \mathrm{~K}$ ), the $H S T$ limits for a blackbody source of a given temperature (black line), the SN 2012Z progenitor system (thin black cross), and the V445 Pup progenitor system (brown cross; F814W magnitude estimated from temperature and $V$-band magnitude). Also plotted are stellar evolution tracks for stars with initial masses of $2,3,4$, and $5 \mathrm{M}_{\odot}$ (Bertelli et al. 2009) and the region predicted for one set of He-star progenitor models from Liu et al (2010) (blue region). The limits shown are the single-band 3- $\sigma$ limits for black bodies. The grey band indicates the additional 1- $\sigma$ distance modulus uncertainty. The distance uncertainty is included in the uncertainties for S1.

the HST images), making a chance alignment somewhat unlikely. Additionally, there are only 53 stars within the same area that are brighter than $\mathrm{S} 1$; there is only a $0.54 \%$ $(4.2 \%)$ chance of being within $1.1 \sigma(3 \sigma)$ of a star at least as bright as S1. Although this latter measurement is an a posteriori statistic, it is helpful for determining the rarity of such stars and what we would have determined for slightly shallower images.

The likelihood of chance alignment is small, but not sufficiently small to completely rule out that scenario. Our above calculation assumes no spatial clustering of stars, which is incorrect. However, it is not possible to precisely determine the necessary correction with the current data. Nonetheless, if $\mathrm{S} 1$ is not part of the SN 2008ha progenitor system, but is located nearby because it was born in the same cluster, this is not a chance coincidence. In that scenario, S1 will have a similar age (and composition) as the SN 2008ha progenitor system. Future observations could possibly differentiate these scenarios.

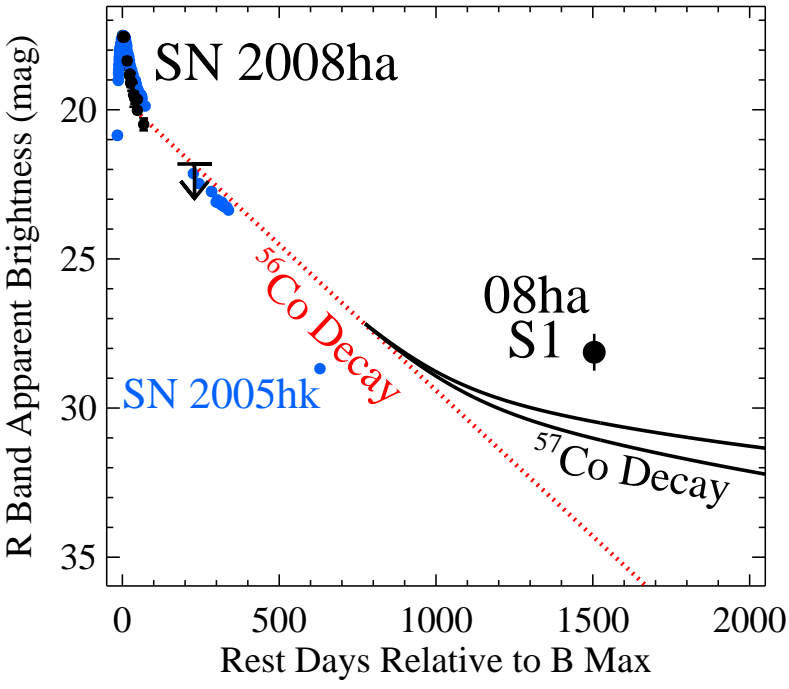

FIG. 3.- $R$-band light curve of SN 2008ha and photometry of S1 (converting our blackbody fit to an $R$ band magnitude). The blue points are the $R$-band light curve of SN 2005hk (Phillips et al. 2007; (McCully et al. 2014a), shifted to match the peak brightness of SN 2008ha. The red-dotted line is the expected light curve extrapolated from the last detection (and is thus an upper limit) if the SN is only powered by ${ }^{56} \mathrm{Co}$ decay. The black solid lines are the model SN Ia light curves from Röpke et al. (2012) that include additional radioactive elements. The two curves represent different explosion mechanisms and thus slightly different nucleosynthetic yields. $\mathrm{S} 1$ is $>2 \mathrm{mag}$ brighter than the expected emission from SN 2008ha at the time of our HST images.

\subsection{Supernova Emission}

SN 2008ha was a very low luminosity event, likely the least luminous hydrogen-deficient SN yet observed. It peaked at $V=17.68 \mathrm{mag}$, corresponding to $M_{V}=$ $-14.17 \mathrm{mag}$, and declined quickly with $\Delta m_{15}(B)=$ $2.17 \mathrm{mag}$ (Foley et al. 2009). SN 2008ha was last detected at $V=20.2 \mathrm{mag} 68$ days after $B$ maximum (Valenti et al. 2009). A later non-detection of $R>$ $22.5 \mathrm{mag}$ at 230 days after $B$ maximum was consistent with ${ }^{56} \mathrm{Co}$ decay extrapolated from the last detection (Foley et al. 2010a), but the true brightness of the SN may have been much fainter. We reproduce the SN 2008ha light curve of Foley et al. (2010a) in Figure 3 . including our observation of S1. The last detection and the non-detection are 8.6 and 5.6 mag brighter than S1 (and roughly the same for our limits), respectively. However, our observation of S1 was obtained 1505 days after $B$ maximum (in the rest frame), and extrapolating the ${ }^{56} \mathrm{Co}$ decay to that time, we expect the $\mathrm{SN}$ to be at least 6.2 mag fainter than $\mathrm{S} 1$.

It is expected that SN light curves should fade slower than the ${ }^{56} \mathrm{Co}$ decay rate at times $\gtrsim 1000$ days after $B$ maximum, when the ${ }^{57} \mathrm{Co}$ decay becomes the dominant contribution to the SN luminosity (Seitenzahl et al.|2009; Röpke et al. 2012). We place the Röpke et al. (2012) model SN Ia light curves (scaled in luminosity to match SN 2008ha) in Figure 3. The models invoke different explosion mechanisms (either violent merger or delayed detonation), which result in somewhat different amounts of particular radioactive nucleosynthetic products and thus slightly different luminosities at very late times. 
The details of the nucleosynthesis may be particularly important for SN 2008ha, but both model light curves are much fainter $(2.3$ and $2.8 \mathrm{mag})$ than $\mathrm{S} 1$ at the time of our observation. In fact, $\mathrm{S} 1$ is brighter than the expected brightness of SN 2008ha at 1000 days, before the other radioactive products are expected to strongly affect the light curve. Since the radioactive decay is exponential, even a doubling of the abundance of these long-lived radioactive species will not dramatically affect the latetime luminosity.

Additionally, S1 is more luminous than SN Iax 2005hk at 630 days after maximum (McCully et al. 2014a), after shifting its peak brightness to match that of SN 2008ha. Unless the SN faded in an unexpected way (or rebrightened), S1 is inconsistent with being emission from the $\mathrm{SN}$. For $\mathrm{S} 1$ to be SN emission, it must have faded by an average of $<1.4$ mag $\mathrm{yr}^{-1}$ from the time of the nondetection.

Any circumstellar dust extinction will only increase the discrepancy between the expected SN luminosity and that of S1.

A final possibility is that $\mathrm{S} 1$ is a light echo of SN 2008ha. This possibility is highly unlikely; there is no indication of dust in front of the SN, S1 is not resolved, and light echoes are usually bluer than the SN.

\subsection{Remnant}

If the explosion fails to unbind the progenitor star, then there will be a gravitationally bound remnant. Such an object has never before been identified, but it is possible that it could be quite luminous.

From early-time spectra, we know that some of the SN 2008ha ejecta is traveling at speeds much greater than the escape velocity of the WD, and Foley et al. (2010a) estimated that there was $0.3 \mathrm{M}_{\odot}$ of unbound material. From mass loss alone, we expect the radius of the WD to change, but not significantly. If the progenitor was a Chandrasekhar-mass WD, it would have lost $\sim 20 \%$ of its mass in the explosion and would have expanded to about twice its radius. At lower masses, the radius of a WD has a weak dependence on its mass, $R \propto M^{-1 / 3}$; a $0.45 \mathrm{M}_{\odot}$ WD losing $0.3 \mathrm{M}_{\odot}$ (67\% of its mass), would only have its radius increase by $44 \%$.

Probably more important to the radius and luminosity of any remnant is the additional energy input directly from the explosion and from radioactive material left in the star. If there is a surviving companion star, then the radius of the remnant is effectively limited by the Roche radius under normal conditions. Once the remnant grows to that radius, mass transfer to the companion will begin. However, the conditions 4 years after the explosion may not be close to an equilibrium; for instance, the remnant may drive a strong opaque wind, which would increase the effective radius beyond the Roche radius, creating a common envelope. This super-Eddington wind could explain the persistent photosphere with low-velocity PCygni profiles seen in the late-time spectra of SNe Iax (Jha et al. 2006; Foley et al. 2013; McCully et al. 2014a).

Blackbody fits to the S1 photometry yield a best-fit temperature of $2100 \pm 500 \mathrm{~K}$ and a radius of $1500 \pm$ $800 \mathrm{R}_{\odot}$, both consistent with a TP-AGB star, although the implied temperature is significantly lower and the implied radius is significantly large than most TP-AGB stars and red supergiants (although perhaps consistent during an extreme mass-loss event). We caution overinterpretation of these values; although S1 was strongly detected in one band, it is only marginally detected in a second band with limits in two other bands. Additionally, a blackbody spectrum may not be appropriate for this source. Furthermore, circumstellar dust may make S1 appear redder and fainter than it really is. However if S1 has minimal circumstellar reddening and is roughly a blackbody, it must be somewhat cool and large.

About $2 \times 10^{46}$ erg of energy is needed to expand a $1 \mathrm{M}_{\odot}$ WD to $\sim 1000 \mathrm{R}_{\odot}$. There was $\sim 2 \times 10^{48} \mathrm{erg}$ of kinetic energy coupled to the ejecta of SN 2008ha (Foley et al. 2009). Therefore, about 1\% of the explosion energy needs to be injected into the star to expand it to our measured radius. Even less energy is needed if only a low-mass envelope expanded to those radii. If the star were to do this, the effective temperature would decrease, and the remnant may appear similar to an TPAGB star.

Jordan et al. (2012) and Kromer et al. (2013) presented single-plume deflagration explosion models for SNe Iax. These models, which do not burn through the entire $\mathrm{WD}$, left $\mathrm{a} \sim 1 \mathrm{M}_{\odot}$ remnant that was polluted with nucleosynthetic ash, and particularly some ${ }^{56} \mathrm{Ni}$. Assuming that there was full $\gamma$-ray trapping in the remnant, Kromer et al. (2013) indicated that the remnant may become more luminous than the SN at $\sim 100$ days after $B$-band maximum. The luminosity of this remnant, however, is tied to the amount of heating from the explosion and the ${ }^{56} \mathrm{Ni}$ in the remnant, making the remnant luminosity only slightly larger than the SN luminosity at late times. The remnant luminosity should decrease slower than that of the SN, but this decay is still larger than observed for S1. The remnant was not resolved in their simulation, but Kromer et al. (2013) expects the remnant to expand and be "puffed up," qualitatively consistent with the large radius inferred for $\mathrm{S} 1$.

\subsection{Companion Star}

M14 discovered the progenitor system for SN 2012Z, another SN Iax, in pre-explosion HST images. For that case, it is impossible for the detected source to be the SN emission or a remnant. Although it is possible that this emission came from an accretion disk around the WD, the best interpretation is that it is the companion star to the WD that exploded. For SN 2008ha, with the deep $H S T$ images obtained after the $\mathrm{SN}$, the interpretation of $\mathrm{S} 1$ is not as clear, but it could be a companion star.

Examining Figure 2, we see that S1 is near the AGB/red supergiant portion of the HR diagram, but is redder than model stellar evolution tracks. The red color of S1 may be caused by newly formed circumstellar dust, but we have no constraint on any circumstellar dust reddening. Using stellar evolution tracks (Bertelli et al. 2009) with a low metallicity $(12+\log [\mathrm{O} / \mathrm{H}]=8.16$, as measured from the nearby H II region; Foley et al. 2009) and ignoring possible circumstellar reddening, S1 has the temperature and luminosity of a TP-AGB star. Because of the scant photometric data for S1, we use the F814W luminosity to determine the possible initial mass range. Since this calculation does not incorporate any color information, we consider all models consistent to within five times the photometric uncertainty of the F814W luminosity of S1 to be consistent. This 
limit includes the possibility of circumstellar reddening of $E(B-V) \leq 0.4 \mathrm{mag}\left(A_{V} \leq 1.2 \mathrm{mag}\right)$. The data are consistent with a TP-AGB star that is in the midst of dramatic mass loss. The luminosity of $\mathrm{S} 1$ is consistent with TP-AGB stars with initial masses of $M>3 \mathrm{M}_{\odot}$ (for $Z=0.004$; Marigo \& Girardi 2007). If there is even more circumstellar reddening, the temperature could change dramatically, and specific conclusions about the nature of S1 would need to be adjusted.

This mass range is consistent with having a $\mathrm{C} / \mathrm{O} \mathrm{WD}$ companion, as is necessary for SN 2008ha (Folev et al. 2010a). Therefore, the TP-AGB explanation makes S1 a viable candidate for a companion star in the SN 2008ha progenitor system.

SNe Iax come from relatively young populations (Foley et al. 2009; Lyman et al. 2013), and thus the stars close to the position of SN 2008ha can constrain the age of the progenitor system. Fitting isochrones to nearby stars (Bertelli et al. 2009; Figure 4), we find a best-fit logarithmic age (in years) of $7.74 \pm 0.09$, corresponding to $t=55_{-10}^{+13} \mathrm{Myr}$. However, if we exclude one of the four nearby stars, we determine a $1-\sigma$ upper limit on the age of 80 Myr. This age is consistent with our mass range of TP-AGB stars for S1.

It is possible, although very unlikely, that SN 2008ha had a massive-star progenitor (Valenti et al. 2009; Moriva et al. 2010). For this scenario, the progenitor must have been a very massive star that had its hydrogen and a significant amount of helium stripped from its outer layers. Any such star would have too short a life to allow a $2-6 \mathrm{M}_{\odot}$ companion to evolve to become a TPAGB star. Therefore, if S1 is the companion star to the SN 2008ha progenitor, it is one more reason that its progenitor could not be a massive star. Furthermore, this scenario requires that the progenitor star, which would necessarily had a very short lifetime $(<10 \mathrm{Myr})$, would be much younger than nearby stars.

$\mathrm{S} 1$ is much redder than the likely companion star for SN $2012 Z$ and the progenitor of V445 Puppis, a Galactic helium nova (Kato \& Hachisu 2003; Woudt et al. 2009). Furthermore, S1 is inconsistent with specific models for a massive helium-burning star companion (Liu et al. 2010), which has been proposed as the progenitor systems for SNe Iax (Foley et al. 2013) and SN 2012Z in particular (M14). If S1 is intrinsically as blue as these other stars, but reddened by circumstellar dust, then S1 must also be much more luminous than these stars. It is unlikely that S1 is a reddened, but intrinsically blue, $\mathrm{He}$ star.

However, only a small portion of the parameter space for WD/He star progenitor systems has been probed. It is possible that $\mathrm{S} 1$ is a He star, but instead of a mainsequence He star, it would be a He red giant, similar in color and luminosity to R Cor Bor. Kato et al. (2008) examined the stellar evolution of He stars to the red giant branch. Their approach was similar to that of Paczyński (1971) and others. Kato et al. (2008) found that single He stars with $M>0.8 \mathrm{M}_{\odot}$ would evolve to become much more luminous and redder, with luminosities consistent (although typically higher) with S1. However, the lowest model temperatures were roughly $4500 \mathrm{~K}$, hotter than S1. Although this model should be investigated further, especially if it is found that $\mathrm{S} 1$ is reddened, the data are currently inconsistent with the models.

Although observations of S1 after the SN are consistent with being a TP-AGB star, the SN may have affected the observational properties of any companion star. For SNe Ia, it is expected that any nearby companion will have mass stripped and will be shock heated (e.g., Marietta et al. 2000; Pan et al. 2012; Shappee et al. 2013); as a result, their luminosity is expected to increase. Pan et al. (2012), Pan et al. (2013), and Liu et al. (2013) specifically looked at Hestar companions, the same companion star suggested for SN 2012Z (McCully et al. 2014b). These studies found that because of their size, the amount of mass stripping was much lower for He star companions than mainsequence or TP-AGB companions, but still suggest that the star will "puff up" and become quite luminous.

None of these studies indicated that the companion star should expand to $\sim 1500 \mathrm{R}_{\odot}$ or become as cool as S1. Moreover, SN 2008ha had an ejecta mass and kinetic energy one and three orders of magnitude less than that of a SN Ia, respectively. Any effect from the SN on the companion star should be less than that of a SN Ia explosion. We therefore think that it is unlikely that any companion star is significantly perturbed, and it is extremely unlikely that S1 is an impacted main-sequence He star, but detailed modeling is required to see the full effects of this scenario.

As seen in Figure 2, S1 has very different physical parameters from the SN $2012 \mathrm{Z}$ companion star. There are five scenarios that are consistent with the observations of SNe 2008ha and 2012Z: (1) S1 is not the companion star of in the SN 2008ha progenitor system and the blue He-star companion has a luminosity below our current limits, (2) S1 was a blue He star and the SN significantly altered its appearance (which we consider unlikely), (3) the blue emission from the SN 2012Z progenitor system was from the WD accretion disk (a super-soft source) and the companion star is red and lower luminosity like S1 (which McCully et al. 2014b consider unlikely), (4) S1 is a He red giant, simply a slightly more evolved version of the companion star for SN 2012Z, or (5) SNe Iax have a variety of progenitor systems.

\section{DISCUSSION AND CONCLUSIONS}

Using HST images obtained 4.1 years after the explosion, we detected a stellar source very close to the position of SN 2008ha, which we call "S1." We determined that this source is unlikely to be a chance superposition, but have not ruled out such a coincidence. We have also shown that the luminosity of $\mathrm{S} 1$ is much higher than the expected luminosity of the SN at the time of the observations, leaving three possibilities for S1: it is not directly related to SN 2008ha, it is the remnant of the WD that produced SN 2008ha, or it is the companion star to the WD that produced SN 2008ha.

Fitting isochrones to stars near the position of SN 2008ha, we have determined that the stars near SN 2008ha have a likely age of $<80 \mathrm{Myr}$. This is consistent with the average value determined for SNe Iax based on their locations relative to $\mathrm{H} \alpha$ emission (30-50 Myr; Lyman et al. 2013).

The photometry of S1 suggests that it is a TP-AGB star with an initial mass of $>3 \mathrm{M}_{\odot}$ (Marigo \& Girardi 2007). This mass range is consistent with being the com- 

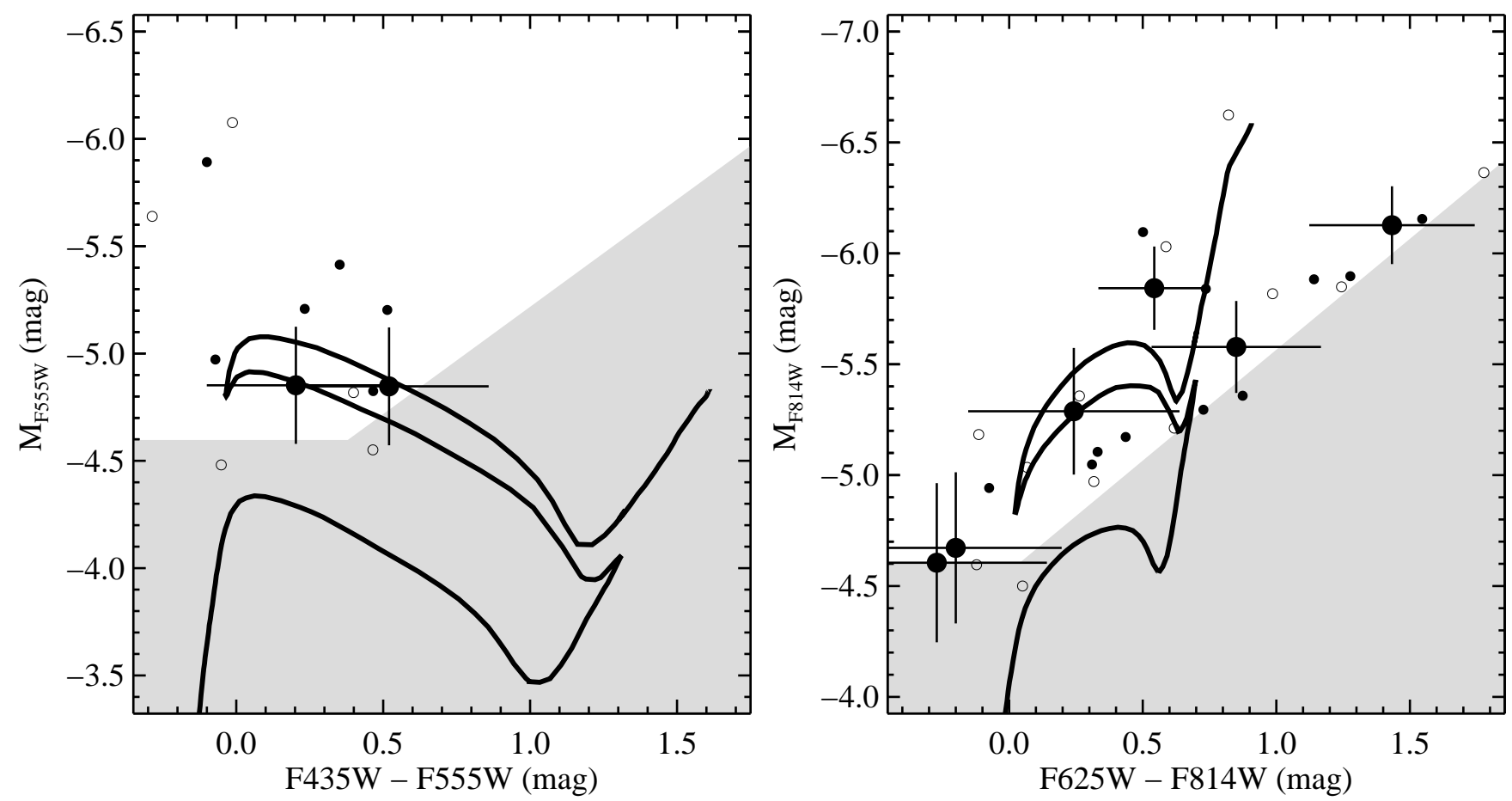

FIG. 4.- Color-magnitude diagrams for the region near SN 2008ha. The large filled (with error bars), small filled, and open circles represent stars within 15,30 , and 45 pixels $\left(0.75,1.5\right.$, and $\left.2.25^{\prime \prime}\right)$ of the position of SN 2008 ha, respectively. The grey area represents magnitudes below our 3- $\sigma$ detection limit. The black curve is a $55 \mathrm{Myr}$ isochrone.

panion of a $\mathrm{C} / \mathrm{O} \mathrm{WD}$ and inconsistent with being the companion of a massive star progenitor.

S1 appears to be too cool to be a He red giant. However, the previous arguments for a He companion star for SN Iax progenitor systems, the properties of the SN 2012Z progenitor system, and the obvious mass transfer triggering by stellar evolution make this option somewhat attractive. Binary evolution models and models of how a SN might change a He red giant star could improve our understanding of the plausibility of this option. Newly formed circumstellar dust may make such a star appear much redder than its intrinsic color.

Regardless, S1 has observational properties very different from the progenitor system for SN $2012 Z$ (M14). It is possible that SNe 2008ha and 2012Z had similar progenitor systems and $\mathrm{S} 1$ has expanded and cooled as the result of the SN impact or that the emission in the SN 2012Z progenitor system is dominated by emission from the accretion disk. However, detailed calculations are required to test these possibilities. If $\mathrm{S} 1$ is the companion star for the SN 2008ha progenitor system and its observational properties have not changed significantly from before the SN explosion, then SNe Iax may have come from a variety of progenitor systems. Perhaps the significant diversity in the properties of SNe Iax (SN 2012Z was 40 times as luminous as SN 2008ha at peak), is related to the diversity of progenitor systems.

It is possible that $\mathrm{S} 1$ is an inflated remnant of the WD. SN 2008ha ejected only $0.3 \mathrm{M}_{\odot}$ of material (Folev et al. 2010a) and therefore likely left a remnant. Models indicate that this remnant should be relatively luminous at late times and "puffed up" (Jordan et al. 2012; Kromer et al. 2013; Fink et al. 2014). S1 has a blackbody radius of $\sim 1500 R_{\odot}$, which is qualitatively consis- tent with these models; moreover, the energy required for this expansion is relatively small compared to the energy of the explosion.

Future observations should be able to further confirm if S1 is the remnant and may rule it out as a companion star. The remnant should vary on years timescales, while it is unlikely that the luminosity of the companion star will change significantly. Eventually, we will build large enough telescopes to obtain a spectrum of S1; if $\mathrm{S} 1$ is indeed the remnant, it will likely have a peculiar spectrum.

Deeper observations at bluer wavelengths will have a better chance of detecting a helium star (the preferred companion star for SN 2012Z) at a position more coincident with SN 2008ha than S1. New HST images could also improve our astrometry by both increasing the $\mathrm{S} / \mathrm{N}$ of $\mathrm{S} 1$ and by measuring additional stars for the astrometric linking. In the future, obtaining deep highresolution images of SNe Iax will improve our ability to constrain progenitor systems and remnants associated with SNe Iax.

S1 represents potentially the second progenitor system of a thermonuclear SN detected, after SN 2012Z. If this is the correct interpretation, it would complicate the Hestar companion scenario of Foley et al. (2013) and require a variety of progenitor systems for SNe Iax. Instead if S1 is the remnant of SN 2008ha, it would be the first detection of such an object. Continued monitoring of S1 will improve our understanding of SN 2008ha and SNe Iax.

\section{Facility: Hubble Space Telescope(ACS)}

We thank the anonymous referee for helpful and in- 
formed comments.

Based on observations made with the NASA/ESA Hubble Space Telescope, obtained at the Space Telescope Science Institute, which is operated by the Association of Universities for Research in Astronomy, Inc., under NASA contract NAS 5-26555. These observations are associated with program GO-12999.

This research at Rutgers University was supported through NASA/HST grant GO-12913.01, and National Science Foundation (NSF) CAREER award AST0847157 to S.W.J. M.D.S. gratefully acknowledges generous support provided by the Danish Agency for Science and Technology and Innovation realized through a Sapere Aude Level 2 grant. This work was supported by the NSF under grants PHY 11-25915 and AST 11-09174.

\section{REFERENCES}

Bertelli, G., Nasi, E., Girardi, L., \& Marigo, P. 2009, A\&A, 508, 355

Chornock, R., Filippenko, A. V., Branch, D., Foley, R. J., Jha, S., \& Li, W. 2006, PASP, 118, 722

Dolphin, A. E. 2000, PASP, 112, 1383

Fink, M., et al. 2014, MNRAS, 438, 1762

Foley, R. J. 2008, Central Bureau Electronic Telegrams, 1576, 2

Foley, R. J., Brown, P. J., Rest, A., Challis, P. J., Kirshner, R. P., \& Wood-Vasey, W. M. 2010a, ApJ, 708, L61

Foley, R. J., et al. 2009, AJ, 138, 376

- 2010b, AJ, 140, 1321

- 2013, ApJ, 767, 57

Iben, Jr., I., \& Tutukov, A. V. 1991, ApJ, 370, 615

Jha, S., Branch, D., Chornock, R., Foley, R. J., Li, W., Swift, B. J., Casebeer, D., \& Filippenko, A. V. 2006, AJ, 132, 189

Jordan, IV, G. C., Perets, H. B., Fisher, R. T., \& van Rossum, D. R. 2012, ApJ, 761, L23

Kato, M., \& Hachisu, I. 2003, ApJ, 598, L107

Kato, M., Hachisu, I., Kiyota, S., \& Saio, H. 2008, ApJ, 684, 1366

Kromer, M., et al. 2013, MNRAS, 429, 2287

Li, W., et al. 2003, PASP, 115, 453

Liu, W.-M., Chen, W.-C., Wang, B., \& Han, Z. W. 2010, A\&A, $523, \mathrm{~A} 3$

Liu, Z.-W., et al. 2013, ApJ, 774, 37

Lyman, J. D., James, P. A., Perets, H. B., Anderson, J. P., Gal-Yam, A., Mazzali, P., \& Percival, S. M. 2013, MNRAS, 434,527

Maoz, D., Mannucci, F., \& Nelemans, G. 2013, ArXiv e-prints, 1312.0628

Marietta, E., Burrows, A., \& Fryxell, B. 2000, ApJS, 128, 615
Marigo, P., \& Girardi, L. 2007, A\&A, 469, 239

Maund, J. R., et al. 2010, ApJ, 722, 1162

McClelland, C. M., et al. 2010, ApJ, 720, 704

McCully, C., et al. 2014a, ApJ, 786, 134

McCully, C., Jha, S. W., Foley, R. J., \& et al. 2014b, Nature, in press.

Milne, P. A., et al. 2010, ApJ, 721, 1627

Moriya, T., Tominaga, N., Tanaka, M., Nomoto, K., Sauer, D. N., Mazzali, P. A., Maeda, K., \& Suzuki, T. 2010, ApJ, 719, 1445

Narayan, G., et al. 2011, ApJ, 731, L11+

Paczyński, B. 1971, Acta Astronomica, 21, 1

Pan, K.-C., Ricker, P. M., \& Taam, R. E. 2012, ApJ, 750, 151

. 2013, ApJ, 773, 49

Phillips, M. M., et al. 2007, PASP, 119, 360

Puckett, T., Moore, C., Newton, J., \& Orff, T. 2008, Central

Bureau Electronic Telegrams, 1567, 1

Röpke, F. K., et al. 2012, ApJ, 750, L19

Schlafly, E. F., \& Finkbeiner, D. P. 2011, ApJ, 737, 103

Schlegel, D. J., Finkbeiner, D. P., \& Davis, M. 1998, ApJ, 500, 525

Seitenzahl, I. R., Taubenberger, S., \& Sim, S. A. 2009, MNRAS, 400, 531

Shappee, B. J., Stanek, K. Z., Pogge, R. W., \& Garnavich, P. M. 2013, ApJ, 762, L5

Stritzinger, M. D., et al. 2014, A\&A, 561, A146

Valenti, S., et al. 2009, Nature, 459, 674

Woudt, P. A., et al. 2009, ApJ, 706, 738 